\title{
In vivo efficiency evaluation of a phage cocktail in controlling severe colibacillosis in confined conditions and experimental poultry houses
}

\author{
Ana Oliveira ${ }^{\mathrm{a}, \mathrm{b}}$, Rui Sereno ${ }^{\mathrm{a}}$, Joana Azeredo ${ }^{\mathrm{b}, *}$

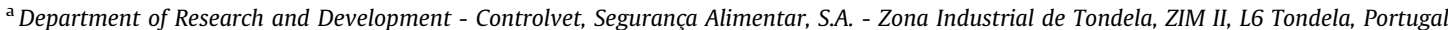 \\ ${ }^{\mathrm{b}}$ IBB - Institute for Biotechnology and Bioengineering, Centre for Biological Engineering, Universidade do Minho, Campus de Gualtar, 4710-057 Braga, Portugal
}

\section{A R T I C L E I N F O}

\section{Article history:}

Received 3 February 2010

Received in revised form 3 May 2010

Accepted 4 May 2010

\section{Keywords:}

Bacteriophages

Therapy

E. coli

Large scale

Poultry

\begin{abstract}
A B S T R A C T
Infections caused by avian pathogenic Escherichia coli (APEC) cause important economic losses to poultry industry.

The studies presented herein, aimed at investigating the in vivo performance of a cocktail of three phages in treating severe respiratory E. coli infections in experimentally contaminated birds and naturally infected flocks.

Three lytic coliphages, phi F78E (Myoviridae), phi F258E (Siphoviridae) and phi F61E (Myoviridae) were combined in a $5.0 \times 10^{7} \mathrm{PFU} / \mathrm{ml}$ cocktail to be used in naturally APEC infected flocks (refractive to antibiotherapy). Experimentally infected birds were treated with phi F78E at two different titres $\left(10^{7} \mathrm{PFU} / \mathrm{ml}\right.$ and $\left.10^{9} \mathrm{PFU} / \mathrm{ml}\right)$. Phage administration was performed orally and by spray, in a single application. The morbidity, mortality and pathology scores were compared with control birds not receiving phage therapy.

The results revealed that the success of phage therapy in experimental rooms was dosage dependent, being $10^{7} \mathrm{PFU} / \mathrm{ml}$ not enough to treat the infected chickens whereas a concentration of $10^{9} \mathrm{PFU} / \mathrm{ml}$ of phi F78E allowed a decrease of $25 \%$ and $43 \%$ in chickens' mortality and morbidity, respectively. In the large scale experiments, the results obtained showed a remarkable efficacy of the low titre phage cocktail $\left(10^{7} \mathrm{PFU} / \mathrm{ml}\right)$ in decreasing the flocks' mortality to levels below $0.5 \%$ in no more than 3 weeks, with no recidivism. Based on the results we can conclude that phage treatment is a valuable alternative to control APEC infections in poultry.
\end{abstract}

(c) 2010 Elsevier B.V. All rights reserved.

\section{Introduction}

Escherichia coli (E. coli) is a commensal bacterium of chickens' intestine. However, some strains, extra-intestinal avian pathogenic E. coli (APEC), are able to trigger invasive infections outside the intestine, namely colibacillosis (Barnes and Gross, 1997; Zhao et al., 2005). This disease is responsible for important economic losses in the poultry industry worldwide, due to lowered production, high treatment costs, carcass rejection at processing and mortality (Vandekerchove et al., 2004; Roy et al., 2006).

\footnotetext{
* Corresponding author. Tel.: +351253 604419; fax: +351253678986. E-mail address: jazeredo@deb.uminho.pt (J. Azeredo).
}

Antibiotic therapy is being used to control this infection, however a significant increase in drug-resistant $E$. coli strains, the restriction to the antibiotic usage implemented by the European Union and the reduced number of new active ingredients entering the market (Scioli et al., 1983; Van den Bogaard et al., 2001) have been limiting its therapeutic effectiveness (Pucci et al., 2004).

Bacteriophages (phages), virus infecting bacteria, have been proposed as valuable candidates as therapeutic agents, based on their capacity to invade and disrupt bacterial metabolism causing the bacteria lysis. Phages are host specific, preventing the destruction of most of the healthy flora, and they are able to multiply exponentially at the site of infection. Phages are harmless for animals, plants and for the environment (Sulakvelidze et al., 2001). 
The efficacy of bacteriophage therapy has been reported by numerous authors. In vivo confined experimental trials have been undertaken to establish the proof of principle of bacteriophage therapy in treating different animals against different types of bacteria (Smith and Huggins, 1983; Smith et al., 1987, Soothill et al., 1988; Soothill, 1992; Sklar and Joerger, 2001; Huff et al., 2002, 2003, 2004; Park and Nakai, 2003; Ronda et al., 2003).

In this work, in vivo trials were performed in order to determine the efficacy of three phages, phi F78E (Myoviridae), phi F258E (Siphoviridae) and phi F61E (Myoviridae), administered orally and by spray, in treating chickens suffering from colibacillosis.

\section{Materials and methods}

\subsection{Isolation of APEC strains}

E. coli strains were isolated from poultry carcasses with colibacillosis, exhibiting typical postmortem lesions as perihepatitis, pericarditis, aerosacculitis and enteritis. Samples of livers, spleen and lungs were collected from carcasses, emulsified in sterile saline solution $(0.85 \% \mathrm{NaCl})$ and $0.1 \mathrm{ml}$ of supernatant was plated on MacConkey agar (Biokar Diagnostics), a selective and differential medium for Gram-negative bacteria. Plates were incubated overnight at $37^{\circ} \mathrm{C}$. As $E$. coli is a lactose fermenter bacteria, the species confirmation of the isolates were conducted by selecting pink-red colonies from the referred media and using API E20 strips, according to manufacturer's instructions (Bio-Merieux). E. coli isolates were stored in Nutrient Broth (Oxoid) with $20 \%$ glycerol, at $-80^{\circ} \mathrm{C}$.

\subsection{Bacteriophage isolation and amplification}

The bacteriophages used in this study, phi F78E, phi F258E and phi F61E, were isolated from samples of sewage, collected randomly from Portuguese poultry houses. Taxonomically, phi F78E and phi F61E seemed to be 1619 type phages belonging to Myoviridae family, yet genetically different, and phi F258E, a T1-like Siphoviridae phage. In vitro tests revealed $70.5 \%$ of lysis effectiveness with a combination of these three phages on 148 APEC strains (Oliveira et al., 2009b). The characteristics of these phages, as well as its broad range of activity, were considered promising and hence were chosen to conduct in vivo phage efficacy experiments in chickens.

Briefly, the isolation assay comprised an overnight incubation $\left(37^{\circ} \mathrm{C}\right)$ of the sewage samples in a 3-4 h culture of the isolated $E$. coli strains, in Luria Bertani (LB) broth, the supernatant centrifugation at $9000 \times \mathrm{g}$ for $10 \mathrm{~min}$ and filtration through $0.22 \mu \mathrm{m}$ membrane filters, and searching for clear zones after spotting the resultant suspension over the respective bacterial strain lawn. The phage replication was performed by inoculating $10 \mathrm{ml}$ of $10^{7} \mathrm{PFU} / \mathrm{ml}$ of each phage suspension in $100 \mathrm{ml}$ culture of the respective host strain, 3-4 h culture in LB broth, followed by an overnight incubation at $37^{\circ} \mathrm{C}$ with shaking $(120 \mathrm{rpm})$. The resultant suspension was centrifuged at $9000 \times \mathrm{g}$ for $10 \mathrm{~min}$, filtered through a $0.22 \mu \mathrm{m}$ membrane and stored at $4{ }^{\circ} \mathrm{C}$.
The phage concentrations were determined based on the plaque assay method described by Adams (1959). A volume of $100 \mu \mathrm{l}$ of successive dilutions of the suspension of each phage, mixed with $100 \mu$ l of the host strain (3-4 h culture) and $3 \mathrm{ml}$ of LB $0.6 \%$ melted agar, was poured onto $1.5 \%$ LB agar plates and incubated overnight at $37{ }^{\circ} \mathrm{C}$.

\subsection{Selection and preparation of the challenge material}

Prior to the phi F78E efficacy trials, in vivo preliminary experiments were conducted in order to select APEC strains able to induce severe but not sudden colibacillosis in chickens under experimental conditions. The strain H839E, serotype 078, was selected to proceed with efficacy trials. The strain, stored in Nutrient Broth (Oxoid) with 20\% glycerol, at $-80^{\circ} \mathrm{C}$, was plated on MacConkey agar, and incubated at $37^{\circ} \mathrm{C}$, overnight. One isolated colony was picked from plates, inoculated in $10 \mathrm{ml}$ of LB broth, and reincubated at $37^{\circ} \mathrm{C}$ for $3 \mathrm{~h}$, just before the beginning of the in vivo efficacy experiments. A sample of this challenge material was taken from the $3 \mathrm{~h}$ grown suspension, to proceed with the determination of the bacterial concentration. For that, successive dilutions of the sample were prepared, in a sterile saline solution $(0.85 \% \mathrm{NaCl})$, and $0.1 \mathrm{ml}$ of each dilution was poured onto LB agar plates and incubated overnight at $37^{\circ} \mathrm{C}$. This procedure was performed in triplicate.

\subsection{Therapeutic phage cocktail composition}

The phage cocktail used in this study was composed of $5.0 \times 10^{7} \mathrm{PFU} / \mathrm{ml}$ of each phage, phi F78E, phi F258E and phi F61E, in LB broth.

\subsection{Welfare, housing and handling}

The in vivo tests performed in confined experimental rooms were designed and conducted in accordance with principles and specific guidelines of animal welfare of the Federation of European Laboratory Animal Science Associations (FELASA) (Van Zutphen et al., 2001), and based on the European Council Directive of 24 November 1986 (86/ $609 / E E C)$ guidelines, regarding the protection of animals used for scientific experimental purposes. In all the designed experiments, there was a great concern to minimize the number of animals used in the experiments.

The in vivo tests were conducted to determine the efficacy of phages in treating chickens with induced colibacillosis. Phages were administered orally, wherein $1 \mathrm{ml}$ of the phage suspension was dispensed into the birds' mouth with the aid of a syringe, and by spray, directly in the beak, through a spray nozzle set to release $1 \mathrm{ml}$ per fine drop scatter (Oliveira et al., 2009a), immediately after chickens have been challenged with an avian pathogenic $E$. coli suspension.

Healthy chickens (Rhode Island Red, acquired from a local poultry house) were housed in batteries, in two experimental rooms, with forced air exhaustion: the birds not exposed to phages were placed in a separate room (named phage-free room) from that of chickens subjected to phage treatment. Temperature and relative humidity 
were measured and controlled during the experiments, in order to ensure the optimal environmental conditions. A 5day acclimation period preceded the challenge. Feed (commercial grower feed) and water were available for ad libitum consumption. The birds were weighed on the day of arrival and at the beginning of the experiment.

The efficacy of the treatments was evaluated based on mortality, morbidity and severity of the colibacillosis lesions (Velkers et al., 2005). Chickens that died during the challenging period, and the ones euthanized at the end of the trial through isoflurane inhalation (Close et al., 1997) were submitted to postmortem examination. The severity of the lesions was evaluated and scored as follows: 1, no macroscopic lesions or thickening and opacity of the inoculated air sac; 2 , non-severe lesions in the internal organs, not interfering in carcass quality; 3, severe and generalised colisepticemia injuries, as fibrinous aerosaculitis, pericarditis and perihepatitis; 4, death before euthanasia. The pathology score of each group was calculated: $\Sigma$ (number of birds with the same score $\times$ score)/total number of birds.

Before the beginning of the trials, chickens were monitored for the presence of other phages active against the challenging $E$. coli strains. For that purpose, feces were collected from each battery, emulsified in LB broth, inoculated in a 3-4 h grown culture of the E. coli strain, and incubated at $37^{\circ} \mathrm{C}$ overnight with shaking (120 rpm). After centrifugation at $9000 \times g$ for $10 \mathrm{~min}$ and filtration through $0.2 \mu \mathrm{m}$, the suspension was spotted on the bacteria lawns (LB agar), incubated at $37{ }^{\circ} \mathrm{C}$ overnight, and checked for clear zones. This procedure was repeated daily in the phage-free room. The screening of phage-sensitive commensal enterobacteria was also performed. Cloacae swabs were collected from chickens, sown in several MacConkey agar plates and incubated at $37^{\circ} \mathrm{C}$. Eight to ten pink colonies were picked from each plate, incubated separately in $10 \mathrm{ml}$ of LB broth at $37^{\circ} \mathrm{C}$ for $3-4 \mathrm{~h}$, and each was spread in a lawn for phage sensitivity test: $10 \mu$ l of phage were dropped on plate bacteria lawns, and incubated at $37^{\circ} \mathrm{C}$ overnight. Plates were then checked for clear zones.

\subsection{In vivo evaluation of phages efficacy to treat colibacillosis}

\subsubsection{Phi F78E high phage titre suspension}

In this trial, the efficacy of a high phi F78E titre suspension to control APEC infections was determined. A total of 28 chickens of 10 -week-old weighing $883.9 \mathrm{~g}$ in average were randomly divided into three groups and placed in batteries. Two groups of 12 birds were challenged with $0.2 \mathrm{ml}$ of a $3 \mathrm{~h}$ grown culture of $5.0 \times 10^{8} \mathrm{CFU} / \mathrm{ml}$ of the APEC H839E, by injection in the left air sac. Immediately after being challenged, one of the groups was treated with a suspension of $1.5 \times 10^{9} \mathrm{PFU} / \mathrm{ml}$ phi F78E, orally $(1 \mathrm{ml})$ and by spray $(1 \mathrm{ml})$.

The other group of 12 birds remained untreated, and was set as the positive control. The negative control, comprised of 4 birds, received sterile LB broth injected in the left air sac.

The euthanasia and postmortem examinations were performed in chickens, 7 days after being challenged with E. coli.
2.6.1.1. Postmortem screening for the presence of host resistant strains. At the postmortem examination of the efficacy trial, infected livers were collected from the phagetreated group. The organs were emulsified, separately, in LB broth. A volume of $0.1 \mathrm{ml}$ of the supernatant was plated in MacConkey agar. Plates were incubated at $37^{\circ} \mathrm{C}$ overnight. Pink-red colonies were picked, inoculated into the same selective media and incubated at $37^{\circ} \mathrm{C}$ overnight; this procedure was repeated three more times.

In each of the experiments, in order to test if the strain isolated from the carcasses remained sensitive to the respective infecting phage, about 10 pink-red colonies were picked from the previously incubated MacConkey agar plates, inoculated separately in 0.6\% LB agar, and poured onto the wells of a 24-well microplate. This procedure was performed in duplicate and a volume of $5 \mu \mathrm{l}$ of phage suspension was dispensed in each bacteriae lawn. The original E. coli strain was used as positive control.

2.6.1.2. Statistical analysis. A two-sided Student's t-test was used, to compare groups with a significance level of $5 \%$ and a statistical power of $90 \%(\alpha=0.05$ and $\pi=0.90)$. The experimental unit was considered to be the chicken. The estimated number of experimental units needed for the trials was obtained based on Beynen et al. (2001) statistical assumptions, in which the sample size was a function of the difference considered meaningful between groups from a physiological point of view, expressed as multiples of the standard deviation that was estimated from an anticipated individual variation $\left(\left(\mu_{1}-\mu_{2}\right) / \sigma\right)$.

The individual variation was previously estimated as $10 \%$ on average, and the meaningful biological difference between groups $(P>0.05)$ was set as $15 \%$, requiring therefore a minimum of 11 experimental units.

\subsubsection{Large scale experiments}

The experimental unit of this experiment was E. coli infected flocks, with high mortality rates even after antibiotic treatment $(n=11)$. Dead chickens in each flock were submitted to postmortem analysis, and after confirmation of death by colisepticemia, through evaluation of macroscopic lesions (perihepatitis, pericarditis, aerosacculitis, enteritis), samples of infected organs - livers, spleen and lungs - were collected from carcasses. Those samples were emulsified $(1: 10(\mathrm{v} / \mathrm{v}))$ in sterile saline solution $(0.85 \% \mathrm{NaCl})$ and $0.1 \mathrm{ml}$ of supernatant was plated on MacConkey agar. Plates were incubated overnight at $37^{\circ} \mathrm{C}$ and pink-red colonies were selected. API E20 strips were used for species confirmation of the isolates.

For each isolated $E$. coli strain, in vitro phage lytic tests were performed, by spotting $10 \mu$ l of the cocktail suspension (phi F61E, phi F78E, and phi F258E) over the respective bacterial lawn on LB agar. Plates were incubated overnight at $37^{\circ} \mathrm{C}$. Clear zones indicated phages in vitro efficacy to lyse the bacteria causing the infection, in the respective flock. In these cases, the phage mixture was administered to all the flock, in a single application. A volume of $500 \mathrm{ml}$ per 10,000 birds was prescribed: half of the dose was diluted in the drinking water to be consumed in half a day and the remaining $250 \mathrm{ml}$ were administered by spray, adding a volume of $500 \mathrm{ml}$ of water per 1000 birds. The 
spray administration was carried out using a commercial manual sprayer set to fine drop deliver, and so that the phage mixture was spread over all the chickens. The water used in this trial was free of disinfectants or other phage inhibitors. Mortality was recorded at the beginning of the trial, and for 3 weeks thereafter.

\section{Results}

\subsection{In vivo evaluation of phi F78E in treating induced colibacillosis}

The microbiological control of the birds at housing revealed no phage particles active against the inoculated host and no phi F78E-sensitive $E$. coli strains in the chickens' feces. The daily phage screening in the phagefree experimental room revealed a total absence of phi F78E phage during trials.

In the experiment, in which the concentration of phage administered was $1.5 \times 10^{9} \mathrm{PFU} / \mathrm{ml}$, the pathology score, the morbidity and the mortality were significantly lower $(P<0.05)$ in the phage-treated group than in the untreated group (H839E) (Fig. 1A). Lesions found in carcasses were also less severe in the phage-treated group (Fig. 1B).

This highly concentrated phage suspension allowed, on average, a $25.0 \%$ decrease in mortality of chickens and $41.7 \%$ in morbidity.

In the negative control group, no chicken died and no lesions were detected in the postmortem analysis.

\subsubsection{Postmortem screening for the presence of host resistant strains}

Results from this test revealed that the E. coli isolated from chickens, receiving the phage preparation, remained sensitive to these phages.

\subsection{Large scale experiments}

E. coli strains isolated from 11 flocks of broiler chickens (Rhode Island Red), 7-week-old on average, were shown, in vitro, to be sensitive to the prepared phage cocktail. Flocks had between 5000 and 10000 birds. The mortality before and after the phage cocktail administration was registered and is presented in Fig. 2. The infection was considered controlled when mortality reached values of $0.5 \%$ or less (usual in healthy flocks). In most cases, when this condition was achieved no further data was collected.

One week after the cocktail administration, the mortality was controlled in five flocks, namely flock number $6,8,9,10$ and 11 ( $\leq 0.5 \%)$. In the following week, flock number 7 achieved the regular levels of mortality, and by the third week, all the flocks except number 5 were controlled for colibacillosis. The exception was related to one case (indicated with an arrow in Fig. 2), in which the mortality decreased consistently since phage administration, from $1.52 \%$ to $0.68 \%$. No recidivism in the mortality rate was observed in any flock until slaughter.

One case study of a broiler flock (Cobb) was also made under the described conditions, and results showed that, besides a mortality decrease during the experiment (from $0.6 \%$ to $0.08 \%$ ), the rejections at slaughter were reduced as well, following phage administration, from $4.8 \%$ to $1.82 \%$ (data not shown).

\section{Discussion}

It has been suggested that phages might be able to reduce the densities and rates of dissemination of the infecting populations of bacteria, to levels at which they may be controlled by the host immune system (Levin and Bull, 2004). Furthermore, by replicating in the infected areas, phages should be able to control localized infections that are relatively inaccessible via the circulatory system as, for example, in the chickens' air sacs. Theoretically, if a bacteriophage reaches the site of a bacterial infection, it is supposed to be effective in eliminating the disease (Sulakvelidze et al., 2001; Cerveny et al., 2002; Sajjad et al., 2004; Huff et al., 2006). These attributes make phages powerful alternatives to antimicrobials. This idea is shared by several phage researchers who reported successful trials, with phages conferring high protection levels against infections (Smith and Huggins, 1983; Smith et al., 1987; Barrow et al., 1998; Sklar and Joerger, 2001; Huff et al., 2002, 2003, 2004, 2005; Park and Nakai, 2003;
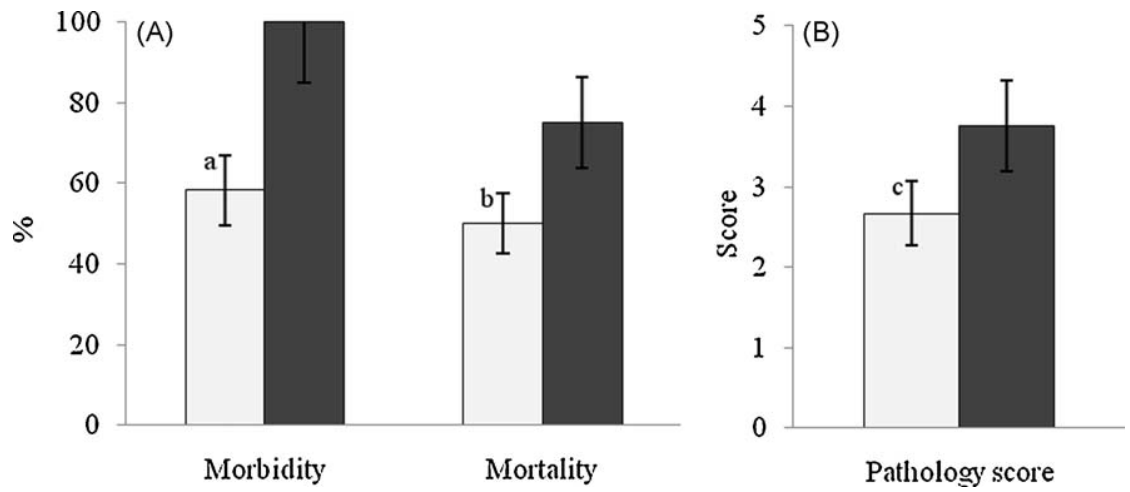

Fig. 1. Morbidity and mortality (\%) (A) and pathology scores (B) obtained for each group of chickens ( $n=12$ ). Groups: phi F78E + H839E ( $\square$ ), challenged with H839E and treated with phi F78E; H839E ( $)$, challenged with H839E. Scores: 1, no injuries; 1-2, non-severe lesions of colibacillosis; 2-3, generalised lesions of colibacillosis; $3-4$, acute colisepticemia. Error bars indicate a meaningful difference of $15 \%$. ${ }^{a}$ Significantly different from morbidity in H839E group; 'bignificantly different from mortality in H839E group; and ${ }^{\mathrm{c}}$ significantly different from pathology scores in H839E group. 


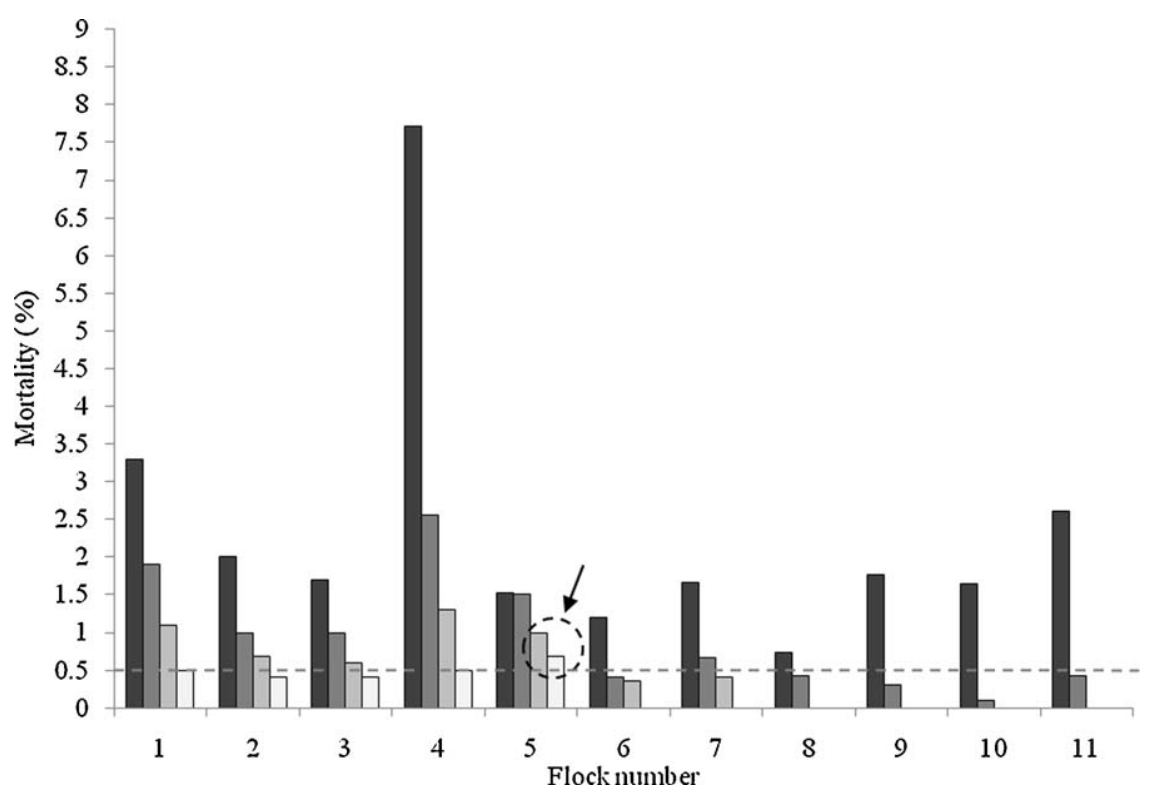

Fig. 2. Mortality rate (\%) measured in $11 \mathrm{E}$. coli naturally infected flocks, previously treated with antibiotics. Records were taken at the phage administration and repeated weekly, for 3 weeks: week before phage administration (

Ronda et al., 2003; Wagenaar et al., 2005). Many authors also recognize benefits in having different phages in the same product, enlarging the lytic spectrum and delaying the occurrence of resistance to phages (Slopek et al., 1987; Smith et al., 1987; Sulakvelidze et al., 2001; Goodridge and Abedon, 2003; Huff et al., 2004; Wagenaar et al., 2005).

In this work, the in vivo efficacy of phages in treating chickens with colibacillosis was evaluated by two different ways: firstly with a high titre phage suspension (phi F78E), conducted in experimental rooms where birds were artificially infected with $E$. coli and secondly in large scale experiments performed on naturally infected flocks with a cocktail comprised by three phages, phi F61E, phi F78E and phi F258E.

Three in vivo studies were previously conducted on the therapeutic efficacy of low titre suspensions, $10^{7} \mathrm{PFU} / \mathrm{ml}$, of phi F61E, phi F78E and phi F258E, administered orally and by spray, to treat APEC challenged chickens. However, there was no efficacy demonstrated, concerning mortality, morbidity and pathological score reduction (data not shown). This was probably based on the low titres of phage used, attending the severe stage of the induced infection in chickens.

In a subsequent study, the therapeutic effectiveness of one of the phages, phi F78E, administered orally and by spray at $10^{9} \mathrm{PFU} / \mathrm{ml}$ was demonstrated, resulting, on average, in a $25.0 \%$ reduction in the mortality and $41.7 \%$ in the morbidity of the treated birds. Results are consistent with most of the published research in this field. Several experiments have been shown that the efficacy of therapy with phages is highly dependent on whether the phage titre administered provides or not a sufficient number of particles to the site of the infection (Huff et al., 2002, 2006).

On the other hand, results obtained in the experiments performed in flocks naturally infected with pathogenic $E$. coli revealed that, when a low titre phage product (three phage cocktail containing $5.0 \times 10^{7} \mathrm{PFU} / \mathrm{ml}$ ) was administered, in a single application, orally and by spray, phages were able to control colibacillosis by avoiding chickens' losses before severe lesions or septicemia occur, allowing a significant mortality reduction in no more than 3 weeks in all flocks, and also a decrease of carcass rejection at slaughter in one tested flock (data not shown).

It is important to stress that, unlike experimental conditions, in naturally-occurring infections the transmission of the infectious agent happens gradually and horizontally from one chicken to another, and birds are not synchronized at the same stage of infection (Huff et al., 2005). So, despite the recognized importance of a high phage concentration for successful therapy (Huff et al., 2006), it is possible that, in naturally-occurring infections in which chickens are in different stages of colibacillosis, a lower titre phage product becomes effective as well, by controlling the earlier stages of the infection and avoiding the progressive transmission in the flock. The product must, nevertheless, be administered as soon as possible, after diagnosis.

The administration of a low phage titre product is advantageous, since it is more feasible to produce large volumes of low concentrated phage suspensions.

Overall, this work demonstrated that the phages under study are able to act therapeutically in early stages of pathogenic E. coli infections, at low doses, when administered orally and by spray.

\section{References}

Adams, M.H., 1959. Bacteriophages. Interscience Publishers, New York, $592 \mathrm{p}$.

Barnes, H.J., Gross, W.B., 1997. Colibacillosis. In: Calnek, B.W., Barnes, H.J., Beard, C.W., McDougald, L.R., Saif, Y.M. (Eds.), Diseases of Poultry. Iowa State University Press, Ames, IA, pp. 131-141. 
Barrow, P., Lovell, M., Berchieri Jr., A., 1998. Use of lytic bacteriophage for control of experimental Escherichia coli septicemia and meningitis in chickens and calves. Clin. Vaccine Immunol. 5, 294-298.

Beynen, A.C., Festing, M.F.M., Monfort, M.A.J., 2001. Design of animal experiments. In: Van Zutphen, L.F.M., Baumans, V., Beynen, A.C. (Eds.), Principles of Laboratory Animal Science. Elsevier, Amsterdam, pp. 219-250.

Cerveny, K.E., DePaola, A., Duckworth, D.H., Gulig, P.A., 2002. Phage therapy of local and systemic disease caused by Vibrio vulnificus in iron-dextran-treated mice. Infect. Immun. 70, 6251-6262.

Close, B., Banister, K., Baumans, V., Bernoth, E.-M., Bromage, N., Bunyan, J., Erhardt, W., Flecknell, P., Gregory, N., Hackbarth, H., Morton, D., Warwick, C., 1997. Recommendations for euthanasia of experimental animals. Part 2. Laboratory Animals. University of Oxford, Oxford, UK, pp. 10-14.

Goodridge, L., Abedon, S.T., 2003. Bacteriophage biocontrol and bioprocessing: application of phage therapy to industry. SIM News $53,254-262$.

Huff, W.E., Huff, G.R., Rath, N.C., Balog, J.M., Donoghue, A.M., 2002 Prevention of Escherichia coli infection in broiler chickens with a bacteriophage aerosol spray. Poult. Sci. 81, 1486-1491.

Huff, W.E., Huff, G.R., Rath, N.C., Balog, J.M., Donoghue, A.M., 2003. Evaluation of aerosol spray and intramuscular injection of bacteriophage to treat an Escherichia coli respiratory infection. Poult. Sci. 82 1108-1112.

Huff, W.E., Huff, G.R., Rath, N.C., Balog, J.M., Donoghue, A.M., 2004 Therapeutic efficacy of bacteriophage and Baytril (enrofloxacin) individually and in combination to treat colibacillosis in broilers. Poult. Sci. 83, 1944-1947.

Huff, W.E., Huff, G.R., Rath, N.C., Balog, J.M., Donoghue, A.M., 2005 Alternatives to antibiotics: utilization of bacteriophage to treat colibacillosis and prevent foodborne pathogens. Poult. Sci. 84, 655-659.

Huff, W.E., Huff, G.R., Rath, N.C., Donoghue, A.M., 2006. Evaluation of the influence of bacteriophage titer on the treatment of colibacillosis in broiler chickens. Poult. Sci. 85, 1373-1377.

Levin, B.R., Bull, J.J., 2004. Population and evolutionary dynamics of phage therapy. Nat. Rev. Microbiol. 2, 166-173.

Oliveira, A., Sereno, R., Nicolau, A., Azeredo, J., 2009a. The Influence of the mode of administration in the dissemination of three coliphages in chickens. Poult. Sci. 88, 728-733.

Oliveira, A., Sillankorva, S., Henriques, A., Quinta, R., Sereno, R., Azeredo, J. $2009 b$. Isolation and characterization of bacteriophages for avian pathogenic E. coli strains. J. Appl. Microbiol. 106, 1919-1927.

Park, S.C., Nakai, T., 2003. Bacteriophage control of Pseudomonas plecoglossicida infection in ayu Plecoglossus altivelis. Dis. Aquat. Organ. 53 33-39.

Pucci, M.J., Bronson, J.J., Barrett, J.F., DenBleyker, K.L., Discotto, L.F., FungTomc, J.C., Ueda, Y., 2004. Antimicrobial evaluation of nocathiacins, thiazole peptide class of antibiotics. Antimicrob. Agents Chemother. 48, 3697-3701.
Ronda, C., Vázquez, M., López, R., 2003. Los bacteriófagos como herramienta para combatir infecciones en Acuicultura. Rev. Aquat. 18, 3-10.

Roy, P., Purushothaman, V., Koteeswaran, A., Dhillon, A.S., 2006. Isolation, characterization, and antimicrobial drug resistance pattern of Escherichia coli isolated from Japanese quail and their environment. J. Appl. Poult. Res. 15, 442-446.

Sajjad, M., Rahman, S.U., Hussain, I., Rasool, M.H., 2004. Application of coliphage lysate: a preliminary trial to treat an experimental Escherichia coli infection in broiler chicken. Int. J. Poult. Sci. 3, 538-542.

Scioli, C., Espostito, S., Anzilotti, G., Pavone, A., Pennucci, C., 1983. Transferable drug resistance in Escherichia coli isolated from antibiotic-fed chickens. Poult. Sci. 62, 382-384.

Sklar, I.B., Joerger, R.D., 2001. Attempts to utilize bacteriophage to combat Salmonella enterica serovar Enteritidis infection in chickens. J. Food Safety. 21, 15-29.

Slopek, S., Weber-Dabrowska, B., Dabrowski, M., Kucharewicz-Krukowska, A., 1987. Results of bacteriophage treatment of suppurative bacterial infections in the years 1981-1986. Arch. Immunol. Ther. Exp. (Warsz.) 35, 569-583.

Smith, H.W., Huggins, M.B., 1983. Effectiveness of phages in treating experimental Escherichia coli diarrhoea in calves, piglets and lambs. J. Gen. Microbiol. 129, 2659-2675.

Smith, H.W., Huggins, M.B., Shaw, K.M., 1987. The control of experimental Escherichia coli diarrhoea in calves by means of bacteriophages. J. Gen. Microbiol. 133, 1111-1126.

Soothill, J.S., 1992. Treatment of experimental infections of mice with bacteriophages. J. Med. Microbiol. 37, 258-261.

Soothill, J.S., Lawrence, J.C., Ayliffe, G.A.J., 1988. The efficacy of phages in the prevention of the destruction of pig skin in vitro by Pseudomonas aeruginosa. Med. Sci. Res. 16, 1287-1288.

Sulakvelidze, A., Alavidze, Z., Morris Jr., J.G., 2001. Bacteriophage therapy Antimicrob. Agents Chemother. 45, 649-659.

Van den Bogaard, A.E., London, N., Driessen, C., Stobberingh, E.E., 2001. Antibiotic resistance of faecal Escherichia coli in poultry, poultry farmers and poultry slaughterers. J. Antimicrob. Chemother. 47, 763-771.

Van Zutphen, L.F.M., Baumans, V., Beynen, A.C., 2001. Principles of laboratory animal science, 2nd Edition. Elsevier, Amsterdam, 428 p.

Vandekerchove, D., Herdt, P.D., Laevens, H., Pasmans, F., 2004. Colibacillosis in caged layer hens: characteristics of the disease and the aetiological agent. Avian Pathol. 33, 117-125.

Velkers, F.C., Te Loo, A.J.H., Madin, F., Van Eck, J.H.H., 2005. Isopathic and pluralist homeopathic treatment of commercial broilers with experimentally induced colibacillosis. Res. Vet. Sci. 78, 77-83.

Wagenaar, J.A., Van Bergen, M.A., Mueller, M.A., Wassenaar, T.M., Carlton, R.M., 2005. Phage therapy reduces Campylobacter jejuni colonization in broilers. Vet. Microbiol. 109, 275-283.

Zhao, S., Maurer, J.J., Hubert, S., DeVillena, J.F., McDermott, P.F., Meng, J., Ayers, S., English, L., White, D.G., 2005. Antimicrobial susceptibility and molecular characterization of avian pathogenic Escherichia coli isolates. Vet. Microbiol. 107, 215-224. 